Published in final edited form as:

Clin Biomech (Bristol, Avon). 2020 February ; 72: 155-160. doi:10.1016/j.clinbiomech.2019.12.019.

\title{
Characteristics of the Gait Initiation Phase in Older Adults with Diabetic Peripheral Neuropathy Compared to Control Older Adults
}

\author{
Gu Eon Kang ${ }^{1}$, He Zhou ${ }^{1}$, Varsha Varghese ${ }^{1}$, Bijan Najafi ${ }^{1,{ }^{*}}$ \\ ${ }^{1}$ Interdisciplinary Consortium on Advanced Motion Performance (iCAMP), Michael E. DeBakey \\ Department of Surgery, Baylor College of Medicine, Houston, Texas, United States
}

\begin{abstract}
Background-Gait is deteriorated in older adults with diabetic peripheral neuropathy; however, too little is known about the gait initiation phase. We aimed to determine if gait initiation variables are more sensitive in identifying the extent to which diabetic peripheral neuropathy impacts gait.

Methods-We examined steps, distance, speed and dynamic balance in the gait initiation phase using a validated algorithm based on wearable sensors in 38 older adults with diabetic peripheral neuropathy and 33 non-diabetic, non-neurologic, non-orthopedic control older adults ( $\geq 65$ years) under single-task and dual-task gait conditions.
\end{abstract}

Findings-During the single-task gait condition, the largest differences between the two groups were found in gait initiation steps and dynamic balance $(66.7 \%$ more steps and $57.2 \%$ poorer balance for the diabetic group; effect size $=1.08$ and 1.11, respectively; all $p<0.05$ ), while gait speed had a medium effect $(10.9 \%$ slower for the diabetic group; effect size $=0.54 ; p<0.05)$. Although gait deteriorated for both groups during the dual-task gait condition compared to the single-task gait condition, effect sizes of the between-group differences remained similar. The differences in gait initiation steps and dynamic balance between the two groups were independent of gait speed.

Interpretation-Gait initiation steps and dynamic balance may be more sensitive than gait speed for detecting gait deterioration due to diabetic peripheral neuropathy. Given the association between gait initiation and risk for fall, our findings suggest that gait initiation variables may be important outcomes for clinical management of diabetic peripheral neuropathy.

\section{Keywords}

Gait initiation phase; Diabetic peripheral neuropathy; Older adults; Step; Distance; Wearable sensors

\footnotetext{
"Corresponding author at: Bijan Najafi, Ph.D., Address: iCAMP, Michael E. DeBakey Department of Surgery, Baylor College of Medicine, 7200 Cambridge St., Houston, Texas, United States 77030, bijan.najafi@bcm.edu.

Publisher's Disclaimer: This is a PDF file of an unedited manuscript that has been accepted for publication. As a service to our customers we are providing this early version of the manuscript. The manuscript will undergo copyediting, typesetting, and review of the resulting proof before it is published in its final form. Please note that during the production process errors may be discovered which could affect the content, and all legal disclaimers that apply to the journal pertain.

Conflict of interest

The authors have no conflict of interest to declare.
} 


\section{Introduction}

Damage to the peripheral nervous system and subsequent decline in somatosensory function, referred to as peripheral neuropathy, is a common complication in diabetes (National Institute of Neurological Disorders and Stroke, 2018). Diabetic peripheral neuropathy (DPN) affects up to half of the population with diabetes (Pop-Busui et al., 2017) and increases the risk of foot ulceration and amputation (Armstrong, Boulton, \& Bus, 2017). Due to the decreased somatosensation, particularly in the lower extremities, DPN is associated with impaired gait performance in older adults, including decreased gait speed and stability (Cavanagh, Derr, Ulbrecht, Maser, \& Orchard, 1992; Courtemanche et al., 1996; Menz, Lord, St George, \& Fitzpatrick, 2004; Zhou et al., 2018). These impairments in gait performance likely lead to an increased risk of falls in older adults with DPN (DeMott, Richardson, Thies, \& Ashton-Miller, 2007; Richardson, Ching, \& Hurvitz, 1992; Schwartz et al., 2002). Most of the evidence in gait impairments in DPN was studied during the steady-state gait phase in which gait speed is maintained relatively constant. Although people typically walk from a static posture, such as an upright standing posture in most situations, little attention has been paid to how gait is initiated from static postures in those with DPN.

The gait initiation phase is the duration in which postural transitions occur from upright standing to steady-state gait (Brunt et al., 1991). In the gait initiation phase, individuals shift their weight from a standing posture towards a stance leg to generate momentum to initiate gait and speed up, to reach steady-state gait speed. It has been reported that an altered gait initiation phase may be associated with the risk of falls (Mbourou, Lajoie, \& Teasdale, 2003). For example, healthy young adults can achieve their steady-state gait speed within two to three steps from an upright standing posture (Miff, Childress, Gard, Meier, \& Hansen, 2005; Miller \& Verstraete, 1996). However, this may not be the case for people at risk for falls, likely due to their postural instability during the gait initiation phase (Hass et al., 2004; Mbourou et al., 2003). In fact, a study reported that people at risk of falling may take more steps and distance to reach their steady-state gait speed (Lindemann et al., 2008).

Despite the association between DPN and risk for fall (DeMott et al., 2007; Richardson et al., 1992; Schwartz et al., 2002), and between falling risk and the gait initiation phase (Lindemann et al., 2008), evidence of characteristics in the gait initiation phase in DPN is sparse. Only a few studies have investigated characteristics of the gait initiation phase in DPN, and these have reported that people with DPN may take between three and six steps to reach a steady-state gait (Gurtej S Grewal et al., 2013; Najafi, Khan, Fleischer, \& Wrobel, 2013). Although the wide age ranges in these studies (i.e., adults $\geq 18$ years) do not necessarily represent older adults, and even though most of their participants were wearing prescribed footwear, such as off-loading shoes and cast shoes, meaning their gait pattern may not necessarily represent their natural gait pattern, these studies showed implications of possible impairments in the gait initiation phase in older adults with DPN. Moreover, DPN is associated with postural instability (Toosizadeh, Mohler, Armstrong, Talal, \& Najafi, 2015), which further supports potential disruption in the gait initiation phase in DPN. 
Another important limitation of the previous gait initiation studies in DPN is a lack of including dual-task gait. It has been reported that people with DPN have a poorer pattern during dual-task gait (i.e., performing a cognitive task during gait) compared to nonneuropathic people (Paul, Ellis, Leese, McFadyen, \& McMurray, 2009), which was caused by less attention on gait during dual-task gait compared to single-task gait (Courtemanche et al., 1996). Similarly, it may be that demanding an additional cognitive task to attention on gait disrupts the gait initiation phase in older adults with DPN, however, this has remained unexplored.

Taken together with previous findings, it may be as important to know the characteristics of the gait initiation phase in older adults with DPN as the steady-state gait phase. The purpose of this study was to investigate the characteristics of the gait initiation phase during singletask gait and dual-task gait in older adults with DPN in comparison to control older adults (CON). Based on gait impairments in DPN (Cavanagh et al., 1992; Courtemanche et al., 1996; Gurtej S Grewal et al., 2013; Menz et al., 2004; Najafi, Khan, et al., 2013), we hypothesized that older adults with DPN would take more steps and longer distance to reach steady-state gait from upright standing posture compared to CON for both single-task and dual-task gait conditions. In addition, we hypothesized that the older adults with DPN would have a slower speed and poorer stability in the gait initiation phase compared to CON for both single-task and dual-task gait conditions.

\section{Methods}

\subsection{Participants}

This study was a secondary analysis of data from a subset of people with DPN and community-dwelling older adults collected previously in three different studies that were reported elsewhere (Gurtej Singh Grewal et al., 2015; Kang, Zahiri, Lepow, Saleem, \& Najafi, 2019; Schwenk et al., 2015). In the previous studies, gait data were collected from a total of 69 people with DPN recruited from outpatient clinics located in Houston (TX, USA) and Tucson (AZ, USA) metropolitan areas, the Baylor College of Medicine (Houston, TX, USA), and the University of Arizona Medical Center (Tucson, AZ, USA) (Gurtej Singh Grewal et al., 2015; Kang et al., 2019). Among them, we included data from people who were aged 65 years and over for the DPN group. We excluded data from people who had a history of neurological conditions, such as stroke and Parkinson's disease. For the CON group, a subset of 125 community-dwelling older adults ( $\geq 65$ years old) was included. The subset did not have a history of diabetes, cancer, orthopedic, or neurological conditions that might affect gait. Consequently, gait data from 38 older adults with DPN and $33 \mathrm{CON}$ were analyzed in this study. All participants were able to walk independently for at least 10 meters. We obtained written informed consent, which was approved by the Institutional Review Boards at the Baylor College of Medicine and the University of Arizona.

\subsection{Clinical assessments}

In order to assess the severity of DPN, we measured vibration perception threshold (VPT) using a Biothesiometer (Bio-Medical Instrument, Newbury, Ohio, United States) as described in previous studies (Kang et al., 2019; Najafi, Crews, \& Wrobel, 2013; Najafi et 
al., 2017; Wang et al., 2019). First, we placed the probe of the Biothesiometer on one of three sites on the plantar surface of the foot: the first and fifth metatarsal heads and heel. We then had gradually increased the electrical vibration from 0 volts until participants began perceiving the vibration. After that, we continued to increase the electrical vibration, then gradually decreased until participants perceived no vibrations. When the difference between the two electrical vibrations (i.e., the vibration level that participants began perceiving and the vibration level that participants stopped perceiving) were less than 2 volts, the larger vibration was considered the VPT value for the site. If the difference was over 2 volts, the procedure was repeated at least three times or until the difference became less than 2 volts. If the difference continued to be over 2 volts, the highest measured value was considered as the VPT value for the site. We repeated this procedure for all the other sites for both feet (i.e., a total of six sites for each participant). VPT $\max$ was the largest VPT value among the six sites (Kang et al., 2019; Najafi, Crews, et al., 2013; Najafi et al., 2017; Wang et al., 2019).

Additionally, we evaluated daily activity level using the Barthel Index of Activities of Daily Living (BI) (Mahoney \& Barthel, 1965), concerns for fall using the Falls Efficacy ScaleInternational (FES-I) (Yardley et al., 2005), and fall history in the past 12 months using selfdisclosure. BI is a 10-item self-administered questionnaire (scores 0-10 per item), and a score of 75 or below was considered moderate to severe disability in daily activities (Supervía et al., 2008). FES-I is a 16-item self-administered questionnaire (scores 1-4 per item), and a score of 28 and over was considered high concern for fall (Delbaere et al., 2010).

\subsection{Gait task}

We evaluated gait performance under single-task and dual-task conditions. Gait performance was evaluated in quiet hallways of Baylor College of Medicine and the University of Arizona Medical Center. For the CON group, all assessments were performed at their own domicile. Each participant began with an upright standing posture with feet apart at their preferred width. Then, each participant began walking forward at their preferred pace. For the single-task gait condition, we asked participants to walk as normally and comfortably as possible. For the dual-task gait condition, participants performed a cognitive task (e.g., counting backward by twos from a random number given by a clinical research coordinator) while walking. Participants were also asked to walk as normally and comfortably as possible for the dual-task gait condition. The order of the gait trials was consistent across all participants (the single-task gait condition followed by the dual-task gait condition), and one gait trial was performed for each task. Participants wore their own walking shoes and walked 12 meters (approximately 40 feet).

We collected gait data using five commercially available inertial sensors that were attached to each participant (LegSys ${ }^{\mathrm{TM}}$, BioSensics, Newton, Massachusetts, United States). The locations of the sensors were the middle of the anterior surface of the thighs bilaterally, the distal end of the anterior surface of the shank bilaterally, and the posterior surface of the lower back. Each sensor consisted of a tri-axial accelerometer, a tri-axial gyroscope, and a magnetometer, and collected linear acceleration and angular velocity of the body segment on which the sensor was attached. The sensor data were obtained with a sampling rate of 100 
Hz. The sensors have been used in previous studies, and the location and algorithms for gait parameters have been validated (Aminian, Najafi, Bula, Leyvraz, \& Robert, 2002; Lindemann et al., 2008; Najafi, Helbostad, Moe-Nilssen, Zijlstra, \& Aminian, 2009; Najafi, Khan, et al., 2013; Najafi, Miller, Jarrett, \& Wrobel, 2010).

\subsection{Data analysis}

In order to calculate gait parameters in the gait initiation phase, we used a previously validated algorithm (Lindemann et al., 2008). Briefly, for each gait task, we first calculated stride velocities of each stride from the $1^{\text {st }}$ stride to the last stride for the entire gait task. The total number of strides were divided into groups of three consecutive strides with neighboring groups have two overlapped strides (e.g., group $1=$ the $1^{\text {st }}, 2^{\text {nd }}$ and $3^{\text {rd }}$ strides; group $2=$ the $2^{\text {nd }}, 3^{\text {rd }}$ and $4^{\text {th }}$ strides; group $3=$ the $3^{\text {rd }}, 4^{\text {th }}$ and $5^{\text {th }}$ strides). In each group, standard deviations of stride velocities among three consecutive strides were calculated. For example, standard deviations of stride velocities in group 1 (among the $1^{\text {st }}, 2^{\text {nd }}$, and $3^{\text {rd }}$ strides), group 2 through the last group were calculated. Then, the median standard deviation for the entire gait task was calculated. The beginning of the steady-state gait phase was defined as the first stride of the groups in which the standard deviation of the stride velocities was below the median standard deviation of all stride velocities. The primary outcome variables were the number of steps and distance to reach steady-state gait, and gait speed and body sway in the mediolateral direction in the gait initiation phase and steadystate gait speed.

\subsection{Statistical analysis}

We compared demographic and clinical characteristics between the DPN and CON groups using Mann-Whitney U tests for age, body-mass index (BMI), BI, FES-I and number of falls in the last 12 months, and chi-square tests for number of women, number of participants who had BI $\leq 75$ (i.e., moderate to severe disability in daily activities), number of participants who had FES-I $\geq 28$ (i.e., high concern for fall), and number of participants who fell in the last 12 months.

For comparing gait parameters, we used the analysis of covariance (ANCOVA) and adjusted for age, sex, BMI, and FES-I. Since most of the gait parameters are highly correlated with gait speed (Kirtley, Whittle, \& Jefferson, 1985), we then added steady-state gait speed as an additional covariate in our statistical model for investigating if an observed significant difference for a gait initiation parameter is mainly due to steady-state gait speed. Nonnormally distributed outcomes (i.e., gait initiation steps, distance, and mediolateral body sway) were ln-transformed before using ANCOVA. We applied Bonferroni correction for ANCOVA. A Bonferroni-adjusted p-value $<0.05$ was considered a significant difference between the DPN and CON groups. For quantifying the differences in gait parameters between the DPN and CON groups, the effect size was calculated using Cohen's d. The magnitude of effect size was classified as follows: Cohen's $d<0.20$ as no noticeable effect, $0.20 \leq$ Cohen's $d<0.50$ as a small effect, $0.50 \leq$ Cohen's $d<0.80$ as a medium effect, Cohen's $d \geq 0.80$ as a large effect (Cohen, 1992). We used SPSS® version 25 (IBM, Armonk, NY, USA) for all statistical analyses. 


\section{Results}

\subsection{Participant characteristics}

We summarized participants' demographic and clinical characteristics in Table 1. Age and BMI were significantly lower and higher, respectively, for the DPN group than for the CON group (all $p<0.05$ ). BI and FES-I were significantly higher and lower, respectively, for the CON group than for the DPN group $(p<0.05$ ). Approximately a quarter of the DPN group fell in the last 12 months. No participants in the CON group fell in the last 12 months. The mean value for $\mathrm{VPT}_{\max }$ in the DPN group was 32.0 volts (SD 14.0).

\subsection{Gait variables}

We summarized steady-state gait speed for each group in Table 2. For both single-task and dual-task gait conditions, the DPN group had $10.9 \%$ and $13.7 \%$ slower gait speed, respectively, compared to the CON group (all $p<0.05$; medium effects).

We summarized characteristics in the gait initiation phase for each group in Table 3. For the single-task gait condition, the mean number of steps and distance to reach the steady-state gait phase were $66.7 \%$ more and $70.4 \%$ longer, respectively, for the DPN group than for the CON group (all $p<0.05$; all large effects) (Figure 1). Gait initiation speed was $12.1 \%$ greater for the CON group than for the DPN group ( $p<0.05$; a medium effect). Mediolateral body sway was $57.2 \%$ larger for the DPN group than for the CON group $(p<0.05$; a large effect).

For the dual-task gait condition, the DPN group took $62.5 \%$ more steps and $86.5 \%$ longer distance to reach the steady-state gait phase compared to the CON group (all $p<0.05$; all large effects). The DPN group had a $12.7 \%$ slower gait speed compared to the CON group ( $p$ $<0.05$; a medium effect). Mediolateral body sway was $67.0 \%$ larger for the DPN group than for the CON group $(p<0.05$; a large effect).

For both single-task and dual-task gait conditions, after accounting for the effects of steadystate gait speed, we found that number of steps, distance, and mediolateral body sway were still significantly greater for the DPN group than for the CON group (all $p<0.05$ ).

\section{Discussion}

This study aimed to characterize the gait initiation phase in older adults with DPN in comparison to CON. We examined the number of steps and distance to reach steady-state gait, and gait speed and mediolateral body sway in the gait initiation phase for the singletask and dual-task gait conditions. All participants were aged 65 years and over. The primary findings were that the DPN group took more, slower, and more unbalanced steps, and a longer distance to reach steady-state gait from an upright standing posture compared to the $\mathrm{CON}$ group. These findings were observed in both the single-task and dual-task gait conditions. Differences in the number of steps, distance and mediolateral body sway were independent of steady-state gait speed. Although some demographic or clinical characteristics such as age, BMI and concern for fall may have affected characteristics of the gait initiation phase, our findings suggest that DPN may have additional negative effects on 
the gait initiation phase in older adults. To our knowledge, this study is the first to investigate the gait initiation phase in older adults with DPN and a higher FES-I.

As hypothesized, the DPN group needed 1.5 times more steps (approximately 4 steps) to reach steady-state gait compared to the CON group (approximately 2.5 steps) for both gait tasks. These steps equated approximately 2 meters for the DPN group, and 1 meter for the CON group to reach the steady-state gait phase. These characteristics were independent of gait speed. It may be that profound somatosensory loss in the feet in the DPN group is a fundamental cause of these differences. Somatosensory function directly affects performance in the first step in the gait initiation phase (Chastan et al., 2010): Disruption in somatosensory function reduces the capacity of postural control during the first step in the gait initiation phase even in healthy adults. Likely due to reduced somatosensory information conveyed from the peripheral nervous system to the central nervous system, the DPN group may have needed one or two more steps for stabilization compared to the CON group. By taking these extra steps, the DPN group may have been able to compensate for postural instability that occurred when generating momentum from an upright standing posture.

Another potential reason for the difference in the number of steps is a concern for fall. In this study, the DPN group had higher concern for fall compared to the CON group. A concern for falls in older adults impairs the first step in the gait initiation phase by increasing duration for postural adjustment when generating momentum (Uemura et al., 2012). Together with the fundamental somatosensory problems related to DPN, the higher concern for fall may have caused a careful gait, resulting in more steps to reach steady-state gait.

Previous studies reported that healthy young adults reach steady-state gait within two or three steps (Miff et al., 2005; Miller \& Verstraete, 1996). In this study, the CON group needed a comparable number of steps. Although the first step in the gait initiation phase may be altered in the CON group compared to healthy young adults (i.e., less capacity to generate forward momentum in the CON group compared to healthy young adults) as suggested by a previous study (Polcyn, Lipsitz, Kerrigan, \& Collins, 1998), our results suggest that the CON group may have compensated the mechanism of speeding up to steady-state gait speed in one or two subsequent steps.

Another important finding in this study was the larger mediolateral body sway for the DPN group than for the CON group. Mediolateral body sway is an important indicator of gait stability, and larger sway indicates a more unbalanced and unstable gait pattern and is associated with the risk of falls during gait or initiating gait (Chen \& Chou, 2017; Lee \& Chou, 2006). Although the DPN group is thought to stabilize balance in the gait initiation phase by taking extra steps compared to the CON group, the results for the mediolateral body sway suggest that older adults with DPN still have unbalanced movement patterns in the gait initiation phase. Our findings were in line with a previous study that reported larger mediolateral body sway during gait on regular and irregular surfaces (Menz et al., 2004). In addition to previous results, our study confirms gait instability in older adults with DPN throughout various phases of gait. 
Slower gait speed for the DPN group compared to the CON group was also consistently found across the gait initiation and steady-state phases. Our results for the gait speed were in line with previous results (Mueller, Minor, Sahrmann, Schaaf, \& Strube, 1994). Together with the results for the larger mediolateral body sway in older adults with DPN, slow speed provides implications of increased falling risk in older adults with DPN in the gait initiation phase as well as the steady-state gait phase.

Notable results from our study were that the greater number of steps, distance, and mediolateral body sway in the gait initiation phase were regardless of gait speed. These results may be clinically meaningful for DPN. Many clinical studies that tested therapeutic efficacy in a treatment on gait performance in DPN focus on improvements in gait speed or stability in the steady-state gait phase (Gurtej Singh Grewal et al., 2015; Morrison, Colberg, Parson, \& Vinik, 2014; Richardson, Thies, DeMott, \& Ashton- Miller, 2004; Wrobel \& Najafi, 2010). However, our results suggest that poor balance control in the gait initiation phase may still exist even after improvements in steady-state gait speed and stability. Thus, it is suggested for clinicians and researchers to take parameters such as the number of steps, distance, and mediolateral body sway in the gait initiation phase into account for clinical management of DPN.

One limitation of this study was that most of our participants in the DPN group had severe symptoms of DPN. It may be that the pattern in the gait initiation phase may be different in people with relatively mild symptoms of DPN. In addition, age, BMI and concern for falls were significantly different between the two groups. Although we addressed these differences as covariates in our statistical model, potential effects from such covariates may still exist. Thus, it is recommended to investigate the gait initiation phase in age-, BMI- and FES-I-matched controls for further confirmation of our results.

\section{Conclusions}

In conclusion, DPN is associated with impairments in the gait initiation phase in older adults who are 65 years and older. Our findings suggest that the number of steps and distance to reach steady-state gait, and mediolateral body sway in the gait initiation phase may also identify deterioration in gait due to DPN. Given the association between the gait initiation phase and risk for fall, our findings also suggest that clinicians and researchers should consider variables in the gait initiation phase when investigating the therapeutic efficacy of treatments for gait performance in older adults with DPN.

\section{Acknowledgments}

This work was supported, in part, by the National Institute of Aging (2R42AG032748-02), and by the National Heart, Lung, and Blood Institute (5T32HL139430-02). However, the funding sources had no role in study design, collection, analysis, and interpretation of data, and writing and submitting this manuscript. The authors thank Ms. Ana Enriquez, Mr. Manuel Gardea and Ms. Maria Noun for their help with data collection.

\section{References}

Aminian K, Najafi B, Bula C, Leyvraz P-F, \& Robert P (2002). Spatio-temporal parameters of gait measured by an ambulatory system using miniature gyroscopes. Journal of biomechanics, 35(5), 689-699. [PubMed: 11955509] 
Armstrong DG, Boulton AJ, \& Bus SA (2017). Diabetic foot ulcers and their recurrence. New England Journal of Medicine, 376(24), 2367-2375. [PubMed: 28614678]

Brunt D, Lafferty MJ, Mckeon A, Goode B, Mulhausen C, \& Polk P (1991). Invariant characteristics of gait initiation. American journal of physical medicine \& rehabilitation, 70(4), 206-212. [PubMed: 1878180]

Cavanagh P, Derr J, Ulbrecht J, Maser R, \& Orchard T (1992). Problems with gait and posture in neuropathic patients with insulin- dependent diabetes mellitus. Diabetic Medicine, 9(5), 469-474. [PubMed: 1611836]

Chastan N, Westby G, du Montcel ST, Do M, Chong R, Agid Y, \& Welter M (2010). Influence of sensory inputs and motor demands on the control of the centre of mass velocity during gait initiation in humans. Neuroscience letters, 469(3), 400-404. [PubMed: 20026383]

Chen T, \& Chou L-S (2017). Effects of muscle strength and balance control on sit-to-walk and turn durations in the timed up and go test. Archives of physical medicine and rehabilitation, 95(12), 2471-2476.

Cohen J (1992). A power primer. Psychological bulletin, 112(1), 155. [PubMed: 19565683]

Courtemanche R, Teasdale N, Boucher P, Fleury M, Lajoie Y, \& Bard C (1996). Gait problems in diabetic neuropathic patients. Archives of physical medicine and rehabilitation, 77(9), 849-855. [PubMed: 8822673]

Delbaere K, Close JC, Mikolaizak AS, Sachdev PS, Brodaty H, \& Lord SR (2010). The falls efficacy scale international (FES-I). A comprehensive longitudinal validation study. Age and ageing, 39(2), 210-216. [PubMed: 20061508]

DeMott TK, Richardson JK, Thies SB, \& Ashton-Miller JA (2007). Falls and gait characteristics among older persons with peripheral neuropathy. American journal ofphysical medicine \& rehabilitation, 86(2), 125-132.

Grewal GS, Bharara M, Menzies R, Talal TK, Armstrong D, \& Najafi B (2013). Diabetic peripheral neuropathy and gait: does footwear modify this association? : SAGE Publications Sage CA: Los Angeles, CA.

Grewal GS, Schwenk M, Lee-Eng J, Parvaneh S, Bharara M, Menzies RA, ... Najafi B (2015). Sensorbased interactive balance training with visual joint movement feedback for improving postural stability in diabetics with peripheral neuropathy: a randomized controlled trial. Gerontology, 61(6), 567-574. [PubMed: 25721132]

Hass CJ, Gregor RJ, Waddell DE, Oliver A, Smith DW, Fleming RP, \& Wolf SL (2004). The influence of Tai Chi training on the center of pressure trajectory during gait initiation in older adults. Archives of physical medicine and rehabilitation, 85(10), 1593-1598. [PubMed: 15468016]

Kang GE, Zahiri M, Lepow B, Saleem N, \& Najafi B (2019). The Effect of Daily Use of Plantar Mechanical Stimulation Through Micro-Mobile Foot Compression Device Installed in Shoe Insoles on Vibration Perception, Gait, and Balance in People With Diabetic Peripheral Neuropathy. Journal of diabetes science and technology, 1932296819839703.

Kirtley C, Whittle MW, \& Jefferson R (1985). Influence of walking speed on gait parameters. Journal of biomedical engineering, 7(4), 282-288. [PubMed: 4057987]

Lee H-J, \& Chou L-S (2006). Detection of gait instability using the center of mass and center of pressure inclination angles. Archives of physical medicine and rehabilitation, 87(4), 569-575. [PubMed: 16571399]

Lindemann U, Najafi B, Zijlstra W, Hauer K, Muche R, Becker C, \& Aminian K (2008). Distance to achieve steady state walking speed in frail elderly persons. Gait \& posture, 27(1), 9196.

Mahoney FI, \& Barthel DW (1965). Functional evaluation: the Barthel Index: a simple index of independence useful in scoring improvement in the rehabilitation of the chronically ill. Maryland state medical journal.

Mbourou GA, Lajoie Y, \& Teasdale N (2003). Step length variability at gait initiation in elderly fallers and non-fallers, and young adults. Gerontology, 49(1), 21-26. [PubMed: 12457046]

Menz HB, Lord SR, St George R, \& Fitzpatrick RC (2004). Walking stability and sensorimotor function in older people with diabetic peripheral neuropathy. Archives of physical medicine and rehabilitation, 85(2), 245-252. [PubMed: 14966709] 
Miff SC, Childress DS, Gard SA, Meier MR, \& Hansen AH (2005). Temporal symmetries during gait initiation and termination in nondisabled ambulators and in people with unilateral transtibial limb loss. Journal of Rehabilitation Research \& Development, 42(2).

Miller CA, \& Verstraete MC (1996). Determination of the step duration of gait initiation using a mechanical energy analysis. Journal of Biomechanics, 29(9), 1195-1199. [PubMed: 8872277]

Morrison S, Colberg SR, Parson HK, \& Vinik AI (2014). Exercise improves gait, reaction time and postural stability in older adults with type 2 diabetes and neuropathy. Journal of Diabetes and its Complications, 28(5), 715-722. [PubMed: 24929798]

Mueller MJ, Minor SD, Sahrmann SA, Schaaf JA, \& Strube MJ (1994). Differences in the gait characteristics of patients with diabetes and peripheral neuropathy compared with age-matched controls. Physical therapy, 74(4), 299-308. [PubMed: 8140143]

Najafi B, Crews RT, \& Wrobel JS (2013). A novel plantar stimulation technology for improving protective sensation and postural control in patients with diabetic peripheral neuropathy: a doubleblinded, randomized study. Gerontology, 59(5), 473-480. [PubMed: 23860103]

Najafi B, Helbostad JL, Moe-Nilssen R, Zijlstra W, \& Aminian K (2009). Does walking strategy in older people change as a function of walking distance? Gait \& posture, 29(2), 261-266. [PubMed: 18952435]

Najafi B, Khan T, Fleischer A, \& Wrobel J (2013). The impact of footwear and walking distance on gait stability in diabetic patients with peripheral neuropathy. Journal of the American Podiatric Medical Association, 103(3), 165-173. [PubMed: 23697719]

Najafi B, Miller D, Jarrett BD, \& Wrobel JS (2010). Does footwear type impact the number of steps required to reach gait steady state?: an innovative look at the impact of foot orthoses on gait initiation. Gait \& posture, 32(1), 29-33. [PubMed: 20362453]

Najafi B, Talal TK, Grewal GS, Menzies R, Armstrong DG, \& Lavery LA (2017). Using plantar electrical stimulation to improve postural balance and plantar sensation among patients with diabetic peripheral neuropathy: a randomized double blinded study. Journal of diabetes science and technology, 11(4), 693-701. [PubMed: 28627217]

National Institute of Neurological Disorders and Stroke. (2018). Peripheral neuropathy fact sheet.

Paul L, Ellis B, Leese G, McFadyen A, \& McMurray B (2009). The effect of a cognitive or motor task on gait parameters of diabetic patients, with and without neuropathy. Diabetic Medicine, 26(3), 234-239. [PubMed: 19317817]

Polcyn AF, Lipsitz LA, Kerrigan DC, \& Collins JJ (1998). Age-related changes in the initiation of gait: degradation of central mechanisms for momentum generation. Archives of physical medicine and rehabilitation, 79(12), 1582-1589. [PubMed: 9862305]

Pop-Busui R, Boulton AJ, Feldman EL, Bril V, Freeman R, Malik RA, ... Ziegler D (2017). Diabetic neuropathy: a position statement by the American Diabetes Association. Diabetes care, 40(1), 136-154. [PubMed: 27999003]

Richardson JK, Ching C, \& Hurvitz EA (1992). The relationship between electromyographically documented peripheral neuropatny and falls. Journal of the American Geriatrics Society, 40(10), 1008-1012. [PubMed: 1328346]

Richardson JK, Thies SB, DeMott TK, \& Ashton- Miller JA (2004). Interventions improve gait regularity in patients with peripheral neuropathy while walking on an irregular surface under low light. Journal of the American Geriatrics Society, 52(4), 510-515. [PubMed: 15066064]

Schwartz AV, Hillier TA, Sellmeyer DE, Resnick HE, Gregg E, Ensrud KE, ... Nevitt MC (2002). Older women with diabetes have a higher risk of falls: a prospective study. Diabetes care, 25(10), 1749-1754. [PubMed: 12351472]

Schwenk M, Mohler J, Wendel C, Fain M, Taylor-Piliae R, \& Najafi B (2015). Wearable sensor-based in-home assessment of gait, balance, and physical activity for discrimination of frailty status: baseline results of the Arizona frailty cohort study. Gerontology, 61(3), 258-267. [PubMed: 25547185]

Supervía A, Aranda D, Marquez MA, Aguirre A, Skaf E, \& Gutierrez J (2008). Predicting length of hospitalisation of elderly patients, using the Barthel Index. Age and ageing, 37(3), 339-342. [PubMed: 18339617] 
Toosizadeh N, Mohler J, Armstrong DG, Talal TK, \& Najafi B (2015). The influence of diabetic peripheral neuropathy on local postural muscle and central sensory feedback balance control. PloS one, 10(8), e0135255. [PubMed: 26258497]

Uemura K, Yamada M, Nagai K, Tanaka B, Mori S, \& Ichihashi N (2012). Fear of falling is associated with prolonged anticipatory postural adjustment during gait initiation under dual-task conditions in older adults. Gait \& posture, 35(2), 282-286. [PubMed: 22024142]

Wang C, Goel R, Rahemi H, Zhang Q, Lepow B, \& Najafi B (2019). Effectiveness of daily use of bilateral custom-made ankle-foot orthoses on balance, fear of falling, and physical activity in older adults: a randomized controlled trial. Gerontology, 65(3), 299-307. [PubMed: 30504728]

Wrobel JS, \& Najafi B (2010). Diabetic foot biomechanics and gait dysfunction: SAGE Publications.

Yardley L, Beyer N, Hauer K, Kempen G, Piot-Ziegler C, \& Todd C (2005). Development and initial validation of the Falls Efficacy Scale-International (FES-I). Age and ageing, 34(6), 614619.

Zhou H, Al-Ali F, Rahemi H, Kulkarni N, Hamad A, Ibrahim R, ... Najafi B (2018) Hemodialysis impact on motor function beyond aging and diabetes_objectively assessing gait and balance by wearable technology. Sensors, 18(11), 3939. 


\section{Highlights}

- We studied gait initiation variables in diabetic neuropathy and control groups.

- The neuropathy group took more gait initiation steps compared to the control group.

- $\quad$ The neuropathy group had poorer dynamic balance compared to the control group.

- $\quad$ Effect size was larger for gait initiation steps and balance than for gait speed.

- Gait initiation variables may better detect gait deterioration than gait speed. 


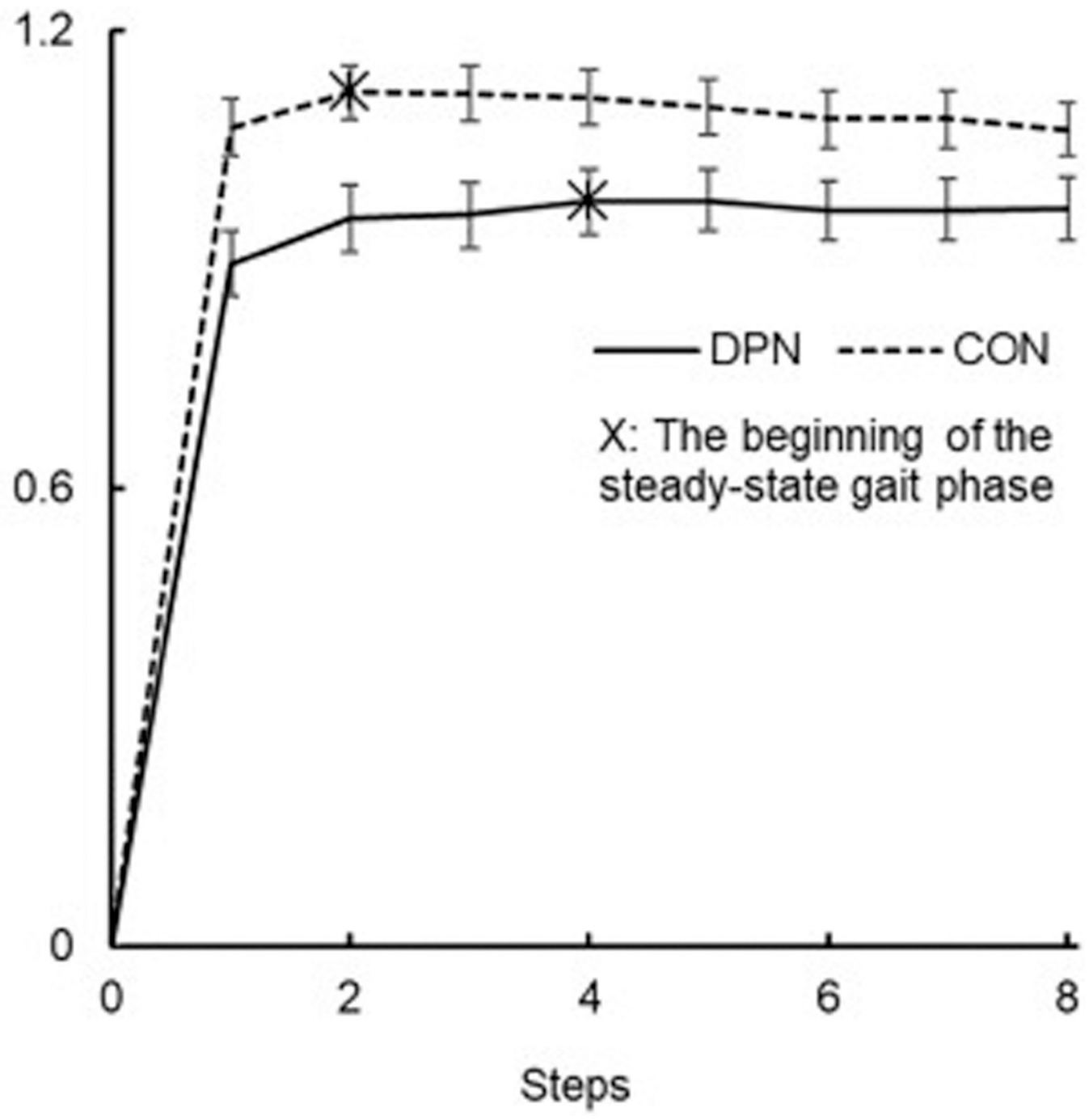

Figure 1.

Mean gait velocity for each step across all participants in the DPN (solid line) and CON (dashed line) groups during single-task gait. The error bars represent standard errors. 
Table 1.

Participants' demographic and clinical characteristics.

\begin{tabular}{llll}
\hline Measures & DPN $(\mathbf{n = 3 8})$ & $\mathbf{C O N}(\mathbf{n = 3 3})$ & $\boldsymbol{P}$-value \\
\hline Women, N & 18 & 20 & 0.265 \\
Age, years & $72.6(5.6)$ & $77.9(8.2)$ & $0.005^{*}$ \\
BMI, kg/m $\mathrm{m}^{2}$ & $31.63(6.07)$ & $27.05(4.23)$ & $0.002^{*}$ \\
Activity level (BI), 0-100 & $95.1(6.0)$ & $95.8(15.7)$ & $0.024^{*}$ \\
$\quad$ Moderate disability in activities (BI $\leq 75), \mathrm{N}$ & 1 & 0 & 0.328 \\
Concern for fall (FES-I), 4-64 & $33.4(14.4)$ & $22.8(8.7)$ & $<0.001^{*}$ \\
$\quad$ High concern for fall (FES-I $\geq 28), \mathrm{N}$ & 20 & 5 & $0.001^{*}$ \\
Number of fallers in the last 12 months, N & 9 & 0 & $<0.001 *$ \\
Number of falls in the last 12 months & $0.7(1.2)$ & $0.0(0.0)$ & $0.001 *$ \\
\hline
\end{tabular}

Note: Values in parentheses represent standard deviations.

Abbreviations denote the following: DPN = Older adults with diabetic peripheral neuropathy; $\mathrm{CON}=\mathrm{Control}$ older adults; $\mathrm{BMI}=\mathrm{Body}-\mathrm{mass}$ index; BI = Barthel Index of Activities of Daily Living; FES-I = Falls Efficacy Scale International.

Asterisks denote significant differences between the DPN and CON groups.

* $P<0.05$. 
Table 2.

Mean values for steady-state gait speed for each group and gait task.

\begin{tabular}{lllll}
\hline Steady-state gait speed, m/s & DPN & CON & $\boldsymbol{P}$-value & Cohen's $\boldsymbol{d}$ \\
\hline Single-task gait & $0.98(0.04)$ & $1.10(0.03)$ & $0.012^{*}$ & $0.54^{\mathrm{M}}$ \\
Dual-task gait & $0.88(0.04)$ & $1.02(0.04)$ & $0.009^{*}$ & $0.58^{\mathrm{M}}$ \\
\hline
\end{tabular}

Note: Values in parentheses represent standard errors.

Abbreviations denote the following: DPN = Older adults with diabetic peripheral neuropathy; CON $=$ Control older adults.

Asterisks denote significant differences between the DPN and CON groups based on ANCOVA tests adjusting for age, body-mass index, sex and FES-I.

* $P<0.05$.

Superscript letter in the Cohen's $d$ column denotes the following: $\mathrm{M}=$ Medium effect size. 
Table 3.

Mean values for gait parameters in the gait initiation phase across all participants for each group and gait task.

\begin{tabular}{lllll}
\hline Gait initiation parameters & DPN & CON & $P$-value & Cohen's $\boldsymbol{l}$ \\
\hline Single-task gait & & & & \\
Number of steps to steady-state gait, steps & $4.0(0.3)$ & $2.4(0.1)$ & $<0.001^{*}$ & $1.08^{\mathrm{L}}$ \\
Distance to steady-state gait, meters & $2.13(0.21)$ & $1.25(0.09)$ & $0.008^{*}$ & $0.88^{\mathrm{L}}$ \\
Gait speed, m/s & $0.99(0.04)$ & $1.11(0.03)$ & $0.018^{*}$ & $0.52^{\mathrm{M}}$ \\
Mediolateral body sway, degrees & $7.01(0.47)$ & $4.46(0.31)$ & $0.001^{*}$ & $1.11^{\mathrm{L}}$ \\
Dual-task gait & & & & \\
Number of steps to steady-state gait, steps & $3.9(0.3)$ & $2.4(0.2)$ & $0.001^{*}$ & $1.05^{\mathrm{L}}$ \\
Distance to steady-state gait, meters & $1.94(0.18)$ & $1.04(0.10)$ & $0.004^{*}$ & $1.04^{\mathrm{L}}$ \\
Gait speed, m/s & $0.89(0.04)$ & $1.02(0.04)$ & $0.025^{*}$ & $0.53^{\mathrm{M}}$ \\
Mediolateral body sway, degrees & $7.18(0.58)$ & $4.30(0.31)$ & $0.001^{*}$ & $1.08^{\mathrm{L}}$ \\
\hline
\end{tabular}

Note: Values in parentheses represent standard errors.

Abbreviations denote the following: DPN = Older adults with diabetic peripheral neuropathy; $\mathrm{CON}=\mathrm{Control}$ older adults.

Asterisks denote significant differences between the DPN and CON groups based on ANCOVA tests adjusting for age, body-mass index, sex and FES-I.

* $P<0.05$.

Superscript letters in the Cohen's $d$ column denote the following: $\mathrm{M}=$ Medium effect size; $\mathrm{L}=$ Large effect size. 